\title{
THE COLLECTIONS OF THREATENED BRYOPHYTES FROM LADOGA KARELIA (RUSSIA) IN FINNISH HERBARIA
}

\section{КОЛЛЕКЦИИ РЕДКИХ И ИСЧЕЗАЮЩИХ ВИДОВ МОХООБРАЗНЫХ ЛАДОЖСКОЙ КАРЕЛИИ (РОССИЯ) В ГЕРБАРИЯХ ФИНЛЯНДИИ}

\author{
HANNA WAHLBERG ${ }^{1}$ \\ ХАННА ВАЛЬБЕРГ ${ }^{1}$
}

Abstract

\begin{abstract}
The study area is the biogeographical province of Ladoga Karelia (Russia) covering the northern and north-western coasts of Lake Ladoga. A list of specimens of threatened bryophytes deposited in Finnish herbaria is given with information of locality and collector. In addition relevant old Finnish literature is reviewed. The list includes 62 mosses, 19 hepatics, 1 hornwort.
\end{abstract}

Резюме

\begin{abstract}
Рассматриваемая территория Ладожской Карелии охватывает северо-западное побережье Ладожского озера. Для нее составлен каталог образцов видов мохообразных, находящихся под угрозой исчезновения, хранящихся в гербариях Финляндии. Приводится также обзор литературы по этому вопросу.
\end{abstract}

\section{INTRODUCTION}

Ladoga Karelia is a biogeographical province covering the northern and north-western coasts of Lake Ladoga. It is mainly a part of the Republic of Karelia, Russia, but the most southern parts of it belong nowadays to the Leningrad Province. Ladoga Karelia consists of 17 old Finnish parishes outlined in the map in Fig. 1 (Anonymous 1938).

"Ladozhskie skhery" is a planned National Park situated in Ladoga Karelia. It consists of costal parts and islands of the former Sortavala, Impilahti and Jaakkima parishes. Information on threatened plants and animals in the area was needed for the action plan of the $\mathrm{Na}$ tional Park. To make the old data preserved in Finnish herbaria available for the authorities, the information of all the specimens of threatened species found was collected and placed in a database. When collecting information for the planned National Park, we treated the whole Ladoga Karelia at the same time.

This article includes the information on the species considered as threatened in the Red Data Book of the Eastern Fennoscandia (Anonymous, 1997) and in the Red Data Book of
Karelia (Maksimov 1995). It is based on the labels of collections located in Finnish herbaria (H, KUO, OULU and TUR). Specimens were not revised in this connection. The aim of this study is to review the bryofloristic work carried out in the Ladoga Karelia by Finnish botanists, and especially to publish the known localities of threatened bryophytes based on the material deposited in Finnish herbaria.

\section{BRYOLOGICAL LITERATURE OF LADOGA KARELIA}

In the late $19^{\text {th }}$ century and in the first half of this century Ladoga Karelia was frequently visited by Finnish botanists. Many of the botanists in that time period had an interest in bryology. Nylander (1852 a, b) was the first to publish information on the bryoflora of Ladoga Karelia. His work was based on his own collections in the area. Next expedition to Karelia was made by Chydenius and Furuhjelm (1859). In their travel diary, they report 8 species of bryophytes from Ladoga Karelia. The most important work including bryophytes in the $19^{\text {th }}$ century was Norrlin's (1878) check-list of the flora of Ladoga Karelia. This work was mainly based on S. O. Lind-

1 - Botalinal Museum of University of Helsinki P. O. Box 47 (Hämeentie 153), Helsinki FIN-00014 Finland (phone (0)9-708-4725; fax (0)9-708-4830; e-mail: hanna.wahlberg@helsinki.fi) 

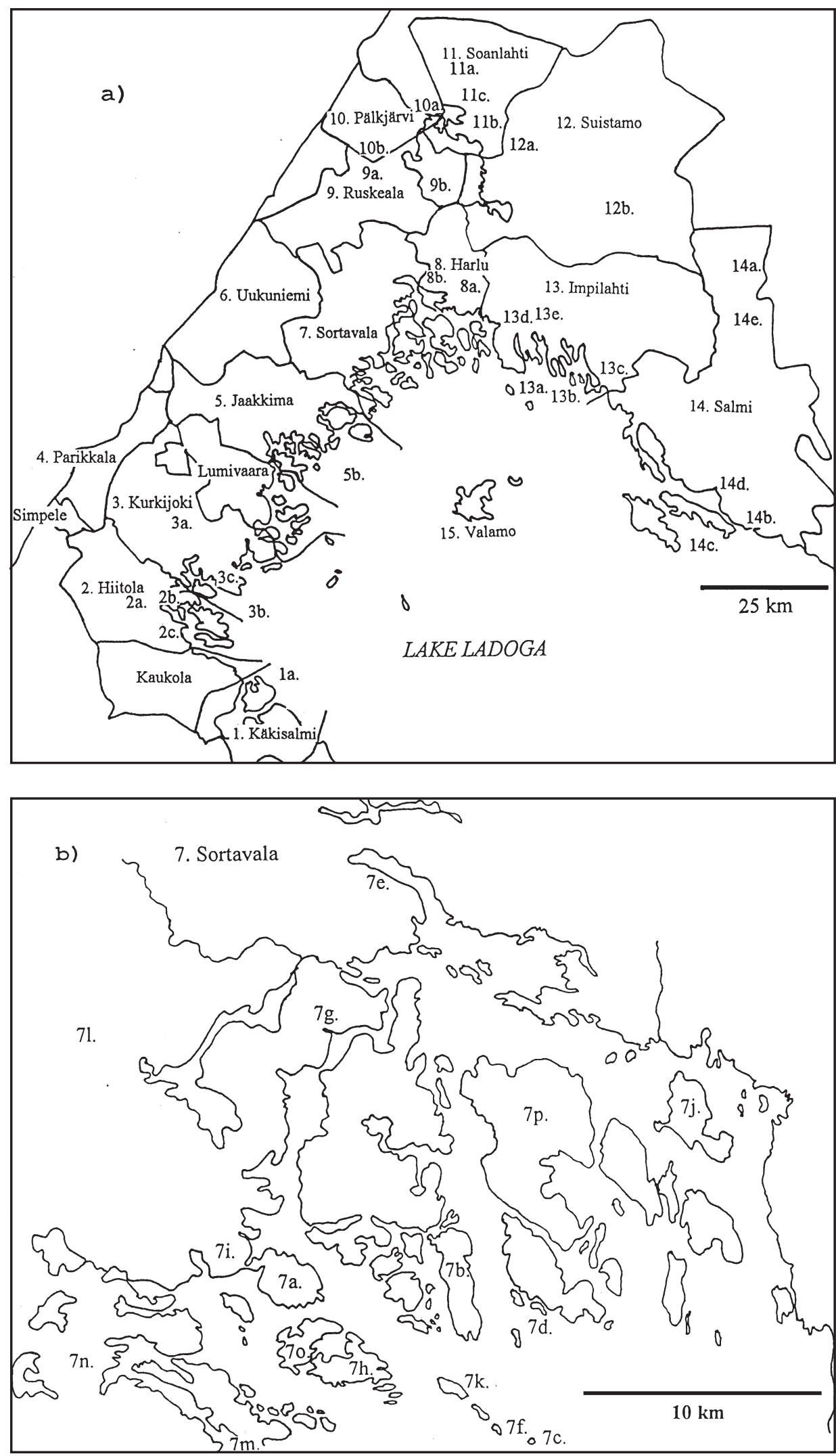
berg's and J. P. Norrlin's collections in the region in the summer of 1874 . In this checklist 162 species of bryophytes are given for Ladoga Karelia. The last major expedition in the last century to Ladoga Karelia was made by H. Hjelt and V. F. Brotherus in 1876 (Hjelt 1881). Brotherus did not publish his collections but Hjelt (1881) reports some of the bryophytes charasteristic to different habitats.

In $1914 \mathrm{~V}$. Pesola made a botanical expedition to the northern parts of Ladoga Karelia (Pesola 1917). He was interested in the effect of limestone on the distribution of plant species and found in his work three new bryophyte species to Ladoga Karelia. M. J. Kotilainen visited Ladoga Karelia several times in the 20's and 30 's and published for instance a description of a botanical excursion to Ladoga Karelia with notes of observed bryophytes (Kotilainen 1931). His major work on bryoflora of the area was his study on the boreal element in the bryoflora of Lagoda Karelia (Kotilainen 1929).

One of the best studied areas belonging to Ladoga Karelia is the former Hiisjärvi Nature Reserve situated in the eastern part of the province, in Salmi (Fig. 1). Brandt (1933) made a survey of the vegetation of the Nature Reserve and Tuomikoski (1935) published a bryophyte flora of the area.

In addition to travel diaries and floras, new findings for the area have been published by many authors. Brotherus (1913) reported Amblystegiella confervoides (Brid.) Loeske (= Platydictya confervoides); Kotilainen (1927) - Cirriphyllum vaucheri Loeske \& Fleisch. (= C. tommasinii); Kotilainen (1935) - Anoectangium compactum Schwaegr. (=A. aestivum), Buch (1938) - Jungermannia tristis Nees (=J. atrovirens), Tuomikoski (1939) - Cryptothallus mirabilis Malmb. and Vaarama (1939) Desmatodon latifolius (Hedw.) Brid. as first records to Ladoga Karelia.
Specimens collected from Ladoga Karelia have been published already in taxonomic revisions and distribution studies. For instances Lindberg (1864) has cited Ladoga Karelian specimens in his study of Timmia species and Tuomikoski (1936) in his study on the mosses of the genus Mnium. Vaarama (1936) studied the distribution of Ricciocarpos natans Corda and many of the specimens cited were collected from Ladoga Karelia. Finnish botanists have traditionally included Russian Karelia when publishing distribution maps of Finnish bryophytes eg. Koponen (1967), Vitikainen (1969), Junnilainen (1977) and Pii ppo (1982).

In the newly published check-list of mosses of Karelia, 352 species are recorded in the area number 12 (Maksimov \& Volkova 1993), which corresponds approximately to the biogeographical province of Ladoga Karelia. Of these 352 species of mosses, 42 species are classified as threatened in the Red Data Book of Karelia (Maksimov 1995). Liverworts and hornworts have not been included in these publications and thus the number of species for Ladoga Karelia remains unknown. On the preliminary list for the coming Red Data Book of Eastern Fennoscandia 69 species of mosses, 1 hornwort and 34 liverworts are classified as threatened in the whole Karelia (Kotiranta \& al. 1997). Altogether, in the Ladoga Karelia there are 62 mosses, 19 liverwors and 1 hornwort considered as thretened in these Red Data books (Kotiranta \& al. 1997, Maksimov 1995).

\section{COLLECTIONS OF THREATENED BRYOPHYTES FROM LADOGA KARELIA.}

This is a list of specimens of threatened bryophytes deposited in Finnish herbaria: H (Botanical Museum, University of Helsinki), KUO (Kuopio Museum of Natural History),

Fig. 1. The localities of collections of threatened bryophytes from Ladoga Karelia. The whole biogeographical province with 17 old Finnish parishes is outlined in the map a. A more detailed picture is given of Sortavala and it's surroundings in map b. 1. Käkisalmi. 1a. Pärnälampi. 2. Hiitola. 2a. Hiitola railway station. 2b. Pekonlahti. 2c. Pukinniemi; Saikanlampi. 3. Kurkijoki. 3a. Haavikko, Ohtijärvi. 3b. Heposaari; Pätäkцnsaari. 3c. Tervu, Vätikkä. 4. Parikkala. 5. Jaakkima. 5a. Puutsalo. 5b. Siikasaari. 6. Uukuniemi. 7. Sortavala. 7a. Haavus; Haukkariutta. 7b. Honkasalo. 7c. Iso-Viro. 7d. Orjatsaari; Kaarnesaari. 7e. Kirjavalahti (Paksuniemi, Hali, Kotomäki,Lahentaus, Louhivuoret, Lakkalampi, Lakkapää, Leppälänniemi, Jamilahti, Vaavalahti).7f. Kotiluoto. 7g. Liikolavuori, Paasovuori, Rausku, Si pilänsalmi. . 7h. Markatsima. 7i. Melloinen, Vuorlahti. 7j. Mäkisalo. 7 k. Mustasaari. 71. Niemikoski. 7m. Pieni Haapasaari. 7n. Rautalahti, Kuokkaniemi. 7o. Tamhanka. 7p. Tulolansaari. 8. Harlu. 8a. Läskelä. 8b. Pцtsцvaara. 9. Ruskeala. 9a. Iso-Selkäsaari. 9b. Marmorilouhos. 10. Pälkjärvi. 10a. Anoniemi, Korkeaniemi. 10b. Matkaselkä, Ruokojärvi, Saarensuo. 11. Soanlahti. 11a. Havuvaara. 11b. Kintsinniemi (dolomiittilouhos). 11c. Korpikallio, Laaja, Vehkavaara, Maitovaara. 12. Suistamo. 12a. Jalovaara. 12b. Leppäsyrjä (Saariselkä, Nykynmäki, Kylänmäki, Säynävaara, Vaaherjoki). 13. Impilahti. 13a. Huunukka. 13b. Majatsaari, Majatsalmi. 13c. Pitkäranta. 13d. Pullinvuori. 13e. Raukkiivuoret. 14. Salmi. 14a. Hiisjärvi. 14b. Karkku. 14c. Lunkula. 14d. Tulema. 14e. Käsnäselkä. 15. Valamo. 
OULU (Botanical Museum, University of Oulu) and TUR (Biology Department, University of Turku). The codes refer to the locations pointed out in Fig. 1. The specimens from KUO and TUR had serial numbers, but the specimens from $\mathrm{H}$ and OULU were numbered while placing the information from the labels to the databank. If the specimens have been confirmed or renamed earlier for a revision or other purposes, the name of the researcher is given before the specimen enumeration. "??" is used for unclear handwriting. Nomenclature of mosses follows Ignatov \& Afonina (1992) and nomenclature of hepatics Konstantinova \& al (1992).

\section{ACKNOWLEGEMENTS}

The study is a part of the project Biodiversity of the northern coast of Lake Ladoga (University of Helsinki, project $n^{\circ}$ 96978501), hosted by the Finnish Museum of Natural History. Financial support by the Ministry of the Environment if gratefully acknowledged. I also want to thank Prof. Timo Koponen for supervising my work.

\section{MOSSES}

Andreaea crassinervia Bruch - 5a. Norrlin, J. P. 1874: H 9. 7n. Huuskonen, A. J. 1943: OULU 380.

Anoectangium aestivum (Hedw.) Mitt. - 11c. Kotilainen, M. 1933: H 306.

Antitrichia curtipendula (Hedw.) Brid.- 3. Juslin, E. 1874: H 14. 7d. Pesola, V. 1914: H 10. 7o. Oesch, L. 1914: H 13. 15. Lindberg, S. O. 1874: H 11. Norrlin, J. P. 1874: H 12.

Atrichum flavisetum Mitt. - 7e. Brotherus, V. F. 1911: H 15, H-Brotherus, V. F. Bryotheca Fennica fasc. 3253.

Barbula unguiculata Hedw. - Brotherus, V. F. 7e. Buch, H. 1922: H 3.

Brachytecium glareosum (Spruce) Schimp. in B. S. G. 9b. Brotherus, V. F. 1903: H 8. Huuskonen, A. J. 1935: TUR 3603, 1936: H 7, 1956: TUR 3595. 12b. Huuskonen. A. J. 1930: OULU 381, 1935: H 6, OULU 366. 1937: OULU 375, TUR 3698. Huuskonen, A. J. \& Huuskonen, I. 1935: OULU 369. Huuskonen, A. J. \& Roivainen, H. 1935: OULU 370. Kotilainen, M. 1926: H 5. Roivainen, H. 1935: H 4.

Bryum knowltonii Barnes - 6. Simming, Th. 1862: H 16.

Bryum stirtonii Bruch et Schimp. in B. S. G. - 10. Brotherus, V. F. 1876: H 17.

Campylium calcareum Crundw. et. Nyh. - 11b. Huuskonen, A. J. 1936: OULU 393. 11. Huuskonen,
A. J. 1936: OULU 394. 12b. Kotilainen, M. 1927: OULU 395.

Campylium elodes (Lindb.) Kindb. - 7e. Backman, H. 1860: H 19, H-SOL 332. Lindberg, S. O. 1874: H 18, H-SOL 333, KUO 386.

Campylium halleri (Hedw.) Lindb. - 7e. Lindberg, S. O. 1874: H 23, H-SOL 331. Norrlin, J. P. 1874: H 22, TUR 5333. 12a. Huuskonen, A. J. 1935: H 21, KUO 1921, OULU 363, 364, TUR 5331, 3412. 1936: H 20, KUO 1925, 1926, OULU 372, TUR 5340, 5334. Kotilainen, M. 1927: H 24, TUR 2336.

Campylium radicale (P.Beauv.) Grout - Lindberg, H. 3. Juslin, E. 1905: H 305.

Cirriphyllum tommasinii (Boul.) Grout - 9b. Huuskonen, A. J. 1934: H 29, OULU 360. 1935: H 30, 31, KUO 2267, OULU 361, 362, TUR 32772, 32782, 32783, 32788, 34186, 34198. 1937: H 32, OULU 373, TUR 7293, 7296, 91225, 91226, 91227, 91228, 91229. Kotilainen, M. 1924: H 27, KUO 2235, 2264, 2269, OULU 382, TUR 7298. 1938: H 28, KUO 2266 TUR 7287. Norrlin, J. P. 1874: H 34. 12b. Huuskonen, A. J. 1935: OULU 367. Kotilainen, M. 1926: H 25. 1930: H 36, TUR 7295. 1931: H 26, TUR 7288, 7292, 7297. Roivainen, H. 1935: H 35. Tuomikoski, R. 19??: H 33.

Coscinodon cribrosus (Hedw.) Spruce - 15. Nylander, W. 1844: H 37.

Desmatodon latifolius (Hedw.) Brid. - 7a. Vaarama, A. 1938: H 38.

Dicranella humilis Ruthe - Brotherus, V. F. 9. Backman, H. 1905: H 307.

Didymodon icmadophilus (Schimp. ex C. Müll.) Saito - 10a. Kotilainen, M. 1927: OULU 389.

Didymodon ridigulus Hedw. - 10a. Brotherus, V. F. 1876: H 40. Linkola, K. 1914: H 39, KUO 785.

Discelium nudum (Dicks.) Brid. - 8a. Pesola, V. 1914: H 42. 14c. Huuskonen, A. J. 1942: OULU 377. 14d. Huuskonen, A. J. 1939: OULU 376. 1942: OULU 378. 1943: OULU 379. 14. Huuskonen, A. J. 1939: H 41, TUR 9598.

Drepanocladus sendtneri (Schimp. ex C.Müll.) Warnst. - Tuomikoski, R. 1940. 9. Brotherus, V. F. \& Buch, H. 1911: H-Brotherus, V. F. Bryotheca Fennica fasc. 3 270. 10b. Waris, H. 1920: TUR 10931-10933, 66373.

Dryptodon patens (Hedw.) Brid. - Buch, H. 1938, Tuomikoski, R. 1940. 7e. Buch, H. 1911: H 57. Lindberg, S. O. 1874: H-SOL 334. Líng, G 1898: OULU 387. Norrlin, J. P. 1874: H 59-61. Poppius, B. 1989: H 56, TUR 41568. 13d. Huuskonen, A. J. 1935: H 55, TUR 13127. 13. Brotherus, V. F. 1876: H 58. 14a. Tuomikoski, R. 1939: H 54.

Encalypta procera Bruch - Horton, D. 1980, 1983. 9. Lindberg, S. O. 1874: H 43, H-SOL 335. 
Encalypta spathulata C.Müll. - Horton, D. 12b. Huuskonen, I. \& Huuskonen, A. J. 1936: H 45. Roivainen, H. 1935: H 44.

Fontinalis hypnoides Hartm. - 1a. Wecksell, J. A. 1907: H 52. 12b. Kotilainen, M. 1939: H 51.

Grimmia anodon Bruch et. Schimp. in B. S. G. Karttunen, K. 1985. 7j. Vainio, E. 1927: TUR 12896. 13c. Brotherus, V. F. 1876: H 53. Brotherus, V. F. \& Hjelt, $\mathrm{Hj}$. 1876: H-SOL 337.

Grimmia elatior Bruch ex. Bals. et. De Not. - Brotherus, V. F. 1918, Auer, A. V. 1936, 1937, Tuomikoski, R. 1946 , Ulvinen, T. 1987. 3b. Räsänen, V \& Laurila, M. 1936: H 82, TUR 12962, 12965. 5a. Norrlin, J. P. 1874: H 80. 5b. Pankakoski, A. 1938: H 71. 7a. Huuskonen, A. J. 1937: H 84. Kari, L. 1923: TUR 12966. Porkka, O. 1923: H 90. Kotilainen, M. 1936: H 68. 7b. Linkola, K. 1914: H 77. Pankakoski, A. 1938: H 73. 7c. Pankakoski, A. 1933: H 75. 1937: H 76. Vaarama, A. 1938: TUR 12963. 7e. Lindberg, S. O. 1874: H-SOL 339. 7f. Hulkkonen, O. 1930: H 89. Kotilainen, M. 1930: H 62, TUR 12964, 12970. Linkola, K. 1929: H 79. Vainio, E. 1927: TUR 12961, 12971. 7g. Lindberg, S. O. 1874: H 87, H-SOL 338. 7h. Pankakoski, A. 1938: H 70. 7j. Vaarama, A. 1938: TUR 12959. Vainio, E. 1927: TUR 12951. 7k. Pesola, V. 1914: H 88. 7m. Pankakoski, A. 1938: H 72. 7o. Linkola, K. 1915: H 78. 9a. Huuskonen, A. J. 1936: H 85, OULU 404. 11c. Brotherus, V. F. 1876: H 65. Huuskonen, A. J. 1936: H 64, OULU 402, 405, 406, TUR 12967, 12969. Kotilainen, M. 1933: H 67, TUR 12968. Roivainen, H. 1935: H 81. 12. Huuskonen, A. J. 1936: H 86. 13a. Pankakoski, A. 1938 : H 74. 13b. Lĺng, G. 1898: H 63. 13c. Kotilainen, M. 1939: H 69. 13d. Brotherus, V. F. 1876: H 83. Huuskonen, A. J. 19??: TUR 12954. Kotilainen, M. 1926: OULU 403, TUR 12953, 12956, 12957. 13e. Kotilainen, M. 1938: H 66.

Grimmia hartmannii Schimp. - Vitikainen, O. 1968. 3a. Juslin, E. 1874: H 110-112. 3. Juslin, E. 1874: H 109, KUO 5091. 7m. Koskimies, A. E. 1927: H 113.

Grimmia ovalis (Hedw.) Lindb. - Auer, A. V. 1937, Tuomikoski, R. 1938, 1945. 2b. Räsänen, V. \& Lau rila, M. 1936: H 102. 3a. Juslin, E. 1874: H 103, 104. 5b. Pankakoski, A. 1938: H 97. 7a. Vaarama, A. 1038: TUR 13081. 7c. Pankakoski, A. 1937: H 100. 7e. Pankakoski, A. 1938: H 99. 7f. Kotilainen, M. 1930: H 91, OULU 388. Pankakoski, A. 1933: H 101. Vainio, E. 1927: TUR 44838. 7g. Lindberg, $S$. O. 1874: H-SOL 340, 341. 7h. Pankakoski, A. 1938: H 98. 7i. Kari, L. 1923: TUR 47903. Linkola, K. 1929: H 96. 7j. Kotilainen, M. 1930: H 92. Kujala, V. 1930: H 95. Vaarama, A. 1938: TUR 13084. 11c. Brotherus, V. F. 1876: H 108. Brotherus, V. F. \& Hjelt, Hj. 1876: H 107. Kotilainen, M. 1933: H 94. 13d. Brotherus, V. F. \& Hjelt, Hj. 1876: H 106. 13e. Kotilainen, M. 1938: H 93. 15. Vaarama,

\section{A. 1938: TUR 13076. ??, ????: H 105}

Grimmia unicolor Hook. in Grev. - Auer, A. V. 5a. Lindberg, S. O. 1874: H-SOL 342. 13d. Kanervo, E. 1926: TUR 13264, 13269, 13272, 41328. Kotilainen, M. 1926: TUR 13275.

Hamatocaulis lapponicus (Norrl.) Hedenäs - Hedenäs, L. 1987. 3c. Juslin, E. 1874: H 115, 116. 3. Juslin. E. 1874: H 117. 11. Lling, G. 1898: H 114.

Homalia besseri Lob. - Buch, H. 1929, Haapasaari, M. \& Fagerstén, R. 1966. 7e. Lindberg, S. O. 1874: H-SOL 343. Norrlin, J. P. 1874: H 119, 121. 10a. Brotherus, V.F. 1876: H 134. Brotherus, V.F. \& Hjelt, Hj. 1876: H 130, 131, TUR 18023. 11c. Brotherus, V. F. 1876: H 136. 1903: H 135. Brotherus, V. F. \& Hjelt, Hj. 1876: H 133. Huuskonen, A. J. 1935: H 129. Kotilainen, M. 1933: H 143. Roivainen, H. 1935: H 122. 12a. Kotilainen, M. 1927: H 139. 12b. Huuskonen, A. J. 1935: H 124-128, OULU 359, TUR 18018, 18021, 19022. Kotilainen, M. 1926 : H 138, 142, OULU 383, TUR 18024. Roivainen, $H$. 1935: H 123. Tuomikoski, R. 1933: H 120, TUR 18015. 13d. Brotherus, V. F. 1876: H 137. Brotherus, V. F. \& Hjelt, Hj. 1876: H 132.

Hymenostylium recurvirostre (Hedw.) Dix. - 7e. Brofeldt, P. 1909: TUR 41666.

Hypnum vaucheri Lesq. - 11c. Brotherus, V. F. 1876 : H 118.

Neckera crispa Hedw. - 2a. Kotilainen, M. 1919: H 155, KUO 7184. 3c. Juslin, E. 1874: H 147. 7e. Koskimies, A. E. 1927: H 146. Laurila, M. 1936: H 150, TUR 18154, 42030. Linkola, K. 1923: TUR 18153. 1927: H 144. Lling, G. 1898: H 145. Norrlin, J. P. 1874: H 151, 152. Parvela, A. 1927: OULU 385. Pesola, V. 1914: H 148. Sahlberg, J. 1872: TUR 18150. Vainio, E. 1927: TUR 18148. 7g. Kari, L. 1923: TUR 18149, 89806. 8b. Tuomikoski, R. 1938: H 154. 11c. Huuskonen, A. J. 1936: OULU 371, TUR 18151. 1937: H 159, KUO 7181, TUR 18152. Roivainen, H. 1935: H 157, KUO 7182. $12 b$. Huuskonen, A. J. 1935: H 158, OULU 368. Kotilainen, M. 1926: H 156, OULU 384. Pesola, V. 1915: H 149. Vainio, E. 1927: TUR 18147. $14 a$. Tuomikoski, R. 1933: H 153.

Neckera pennata Hedw. - 5a. Lindberg, S. O. 1874: H 162, H-SOL 344. 7e. Syrjänen, K., Laaka, S. \& Virtanen, R. 1991: TUR 89901, 89902. 7g. Hidén, I. 1923: H 161. 7. Syrjänen, K., Laaka, S. \& Virtanen, R. 1991: TUR 89900. 11. Líng, G. 1899: H 160. 15. Norrlin, J. P. 1864: H 163.

Orthothecium chryseon (Schwaegr. ex. Schultes) Schimp. in B. S. G. - 7e. Vainio, E. 1926: TUR 18769.

Orthotrichum cupulatum Brid. - 10a. Brotherus, $V$. F. 1876: H 164. 
Orthotrichum pallens Bruch ex. Brid. - Auer, A. V. 1941. 7e. Lindberg, S. O. 1874: H-SOL 345. 7j. Vainio, E 1923: TUR 18972.

Orthotrichum urnigerum Myr. - Schimper. 7. Nylander, W. 1850: H 165.

Philonotis arnellii Husn. - 5a. Lindberg, S. O. 1874: H 174, H-SOL 347. 7e. Lindberg, S. O. 1874: HSOL 346.

Philonotis fontana (Hedw.) Brid. var. falcata (Hook.) Brid. - Field, J. H. 1974. 1. Líng, G. 1897: H 167. 8b. Tuomikoski, R: 1938: H 166. 12b. Huuskonen, A. J. 1935: H 171. 1936: H 170, TUR 19599, 19601. 1938: OULU 398. Huuskonen, A. J. \& Roivainen, H. 1935: OULU 399. Roivainen, $H$. 1935: H 169. 15. Vaarama, A. 1938: H 168.

Physcomitrium sphaericum (Ludw.) Brid. - Buch, H., Karttunen, K. 3. Brotherus, V. F. \& Hjelt, Hj. 1876: H 173, H-SOL 353. 7g. Huuskonen, A. J. 1937: H 172.

Plagiomnium affine (Bland.) T. Kop. - Koponen, T. 1966. 12c. Roivainen, H. 1935: H 176. 12. Roivainen, H. 1935: H 175.

Plagiomnium drummondii (Bruch et. Schimp.) T.Kop. - Koponen, T. 1965, Fagerstén, R. 1979. 7e. Lindberg, S. O. 1874: H 181-183, H-SOL 350-352. Norrlin, J. P. 1874: H 177, 178. 7k. Tuomikoski, R. 1938: H 185187. 10. Brotherus, V. F. 1876: H 180. 14a. Brandt, A. 1931: H 179. Tuomikoski, R. 1934: H 184.

Platydictya confervoides (Brid.) Crum - 7e. Brotherus, V. F. 1911: H-Brotherus, V. F. Bryotheca Fennica fasc. 3 264. Buch, H. 1911: H 1, 2, OULU 358, TUR 267, 276.

Platygyrium repens (Brid.) Schimp. in B. S. G. Auer, A. V. 1936. 2c. Laurila, M. 1935: H 188, TUR 20642, 20644. 3. Juslin, E. 1874: H 189. 7e Brotherus, V. F. 1911: H 193. Lindberg, S. O. 1874: H 190, H-SOL 349. 9. Brotherus, V. F. 1876: H 192. 10a. Brotherus, V. F. 1876: H 194. 10 Brotherus, V. F. \& Hjelt, Hj. 1876: H 191.

Pohlia camptotrachela (Ren. et. Card.) Broth. - Buch, H. 1905. 6. ?? 1862: H 195. 9. Simming, Th. 1862: h 196.

Polytrichum formosum Hedw. - Buch, H. 1932. 3c. Juslin, E. 1874: H 203. 3. Juslin, E. 1874: H 204. 7e. Koskimies, A. E. 1927: H 200, Parvela, A. 1927: OULU 396, Sahlberg, U. 1902: H 197. 7d. Oesch, L. 1914: TUR 22152. 7g. Waris, H. 1924: TUR 22767. 7. Lindberg, S. O. 1874: H-SOL 348. Söyrinki, N. 1929: OULU 397. 10. Brotherus, V. F. 1876: H 202. 11. Líng, G. 1898: H 205-207. 13c. Kotilainen, M. 1939: H 199, TUR 96183. 13. Koponen, J. 1905: H 198. 14a. Tuomikoski, R. 1932: H 201.

Pseudotaxiphyllum elegans (Brid.) Iwats. - 12. Huuskonen, A. J. 1935: H 209, OULU 365. 14a. Tuomikoski, R. 1934: H 208.
Racomitrium aquaticum (Brid. ex. Schrad.) Brid. 12b. Huuskonen, A. J. 1936: KUO 8898, TUR 23395 .

Racomitrium heterostichum (Hedw.) Brid. - 7e. Norrlin, J. P. 1874: H 210, H-SOL 357. ?? 1874: H 211. 15. ?? ????: H 212.

Rhabdoweisia fugax (Hedw.) Bruch et. Schimp. in B: S. G. - 12b. Huuskonen, A. J. 1936: H 213.

Rhynchostegium riparioides (Hedw.) C.Jens. Buch, H. 1929. 11. Cederhvarf, R. 1902: H 214.

Schistidium flaccidum (De Not.) Lindb. - 13d. Brotherus, V. F. 1876: KUO 9550. Brotherus, V. F. \& Hjelt. Hj. 1876: H 308.

Seligeria campylopoda Kindb. in Macoun $-\cdot$ Gos, L. 1992. 12b. Kotilainen, M. 1927: H 216. Roivainen, H. 1935: H 215.

Seligeria donniana (Sm.) C. Müll. - Gos, L. 1992. 9b. Huuskonen, A. J. 1937: H 219, OULU 374, TUR 25041, 25049. Norrlin, J. P. 1874: H 217. 9. Lindberg, S. O. 1874: H-SOL 356, Simming, Th. 1862: H 218, H-SOL 355. 10a. Brotherus, V. F. 1876: H 225. Brotherus, V. F. \& Hjelt, Hj. 1876: H 226. 11c. Roivainen, H. 1935: H 223. 12c. Huuskonen, A. J. 1935: H 220. Huuskonen, A. J. \& Roivainen, H. 1935: TUR 25046. Kotilainen, M. 1927: H 222. 1938: H 221. Roivainen, H. 1935: H 224.

Seligeria recurvata (Hedw.) Bruch et. Schimp. in B. S. G. - Vitt, D. 1976, Gos, L. 1992. 9. Brotherus, V. F. 1904: H-Brotherus, V. F. Bryotheca Fennica fasc. 15. Nylander, W 1842: H-SOL 354. 11b. Huuskonen, A. J. 1936: TUR 25059, 25062. 12b. Huuskonen, A. J. 1935: H 227, TUR 25058. 1936: TUR 25060, 25063.

Sphagnum molle Sull. - Isoviita, P. 1963. 14a. Tuomikoski, R. 1939: H 234.

Splachnum vasculosum Hedw. - 1. Lindberg, H. 1897: H 229. 6. Simming, Th. 1861: H 228.

Trematodon ambiguus (Hedw.) Hornsch. - 6. Simming, Th. 1861: H 230. 12b. Kotilainen, M. 1926: H 231, OULU 400, TUR 29277. 1928: H 232, 233. 14. Huuskonen, A. J. 1936: OULU 401.

Ulota hutchinsiae (Sm.) Hammar - Piippo,S.1981. 2c. Laurila, M. 1935: H 309, TUR 29395. 3. Juslin, E. 1905: H 310.

Zygodon viridissimus (Dicks.) Brid. - Auer, A. V. 1937, Virtanen, R. \& Ohenoja, M. 1992. 11c. Kotilainen, M. 1933: TUR 29597.

\section{HEPATICS AND HORNWORTS}

Anthoceros agrestis Paton - 6. Simming, Th. 1867: H 236.

Arnellia fennica (Gott.) Lindb. - Brotherus, V. F., Buch, H. 1928, 1936. 12b. Huuskonen, A. J. 1935: H 240-244. 1936: H 239. Kotilainen, M. 1926: H 249. 
1927: H 248. 1928: H 252, TUR 30041. 1930: H 246, 247, 251, TUR 30047. 1931: H 250. Pesola, V. 1915: H 245. Tuomikoski, R. 1933: H 237, TUR 30045. Waris, H. 1922: TUR 30043. 14a. Tuomikoski, R. 1933: Н 238.

Calypogeia suecica (H. Arnell et J. Perss.) - Buch, H. 1928, 1935. 7e. Líng, G. 1898: H 254. 14a. Tuomi koski, R. 1934: H 253, TUR 30863, 30864.

Cephaloziella elachista (Jack ex Gott. et Rabenh.) - Buch, H. 1934. 14b. Paasio, J. 1934: H 255.

Conocephalum conicum (L.) Und. - Auer, A. V. 7e. Buch, H. 1911: H 264. Hidén, I. 1923: H 266. Kari, L. 1923: TUR 31447, 31448. Lindberg, S. O. 1874 : H 265, H-SOL 312.

Cryptothallus mirabilis Malmb. - v. Malmborg, S. 12b. Tuomikoski, R. 1936: H 267.

Crossogyna autumnalis (DC.) Schljak. - Grolle, R. 1969. 7e. Lindberg, S. O. 1874: H 271, H-SOL 313. 14a. Tuomikoski, R. 1934: H 269, 270, TUR 32139.

Dichiton integerrimum (Lindb.)Buch - 5a. Lindberg, S. O. 1874: H-SOL 311.

Frullania tamarisci (L.) Dum. - 15. Norrlin, J. P. 1874: H 268.

Leiocolea alpestris (F. Web.) Isov. - 3. Juslin, E. 1874: H-SOL 314. 7e. Lindberg, S. O. 1874: HSOL 316, 317.

Liochlaena lanceolata Nees - Lindberg, S. O., Buch, H., Auer, A. V. 3. Juslin, E. 1874: H 277. 4. Sahlberg, J. 1878: TUR 32161. 9. Buch, H. 1911: H 272. 11a. Huuskonen, A. J. 1937: H 273. 13b. Koponen, J. 1912: H 275. 14a. Tuomikoski, R. 1934: H 274. 15. Juslin, E. 1874: H 276.
Lophozia ascendens (Warnst.) Schust. - 14a. Tuomikoski, R. 1934: H 278-282, TUR 32645.

Mannia fragrans (Balb.) Frye et Clark - Buch, H. 1944. 7j. Vainio, E. 1923: TUR 31834. 11c. Brotherus, V. F. 1876: H 285. 13d. Brotherus, V. F. 1876: H 283, 284.

Marsupella sparsifolia (Lindb.) Dum. - Buch, H. 5a. Norrlin, J. P. 1874: H 286. 14a. Tuomikoski, R. 1934: H 287.

Marsupella sphacelata (Gieseke ex Lindenb.) Dum. Buch, H. 1941, Grolle, R. 1966. 5a. Norrlin, J. P. 1874: H 288, H-SOL 318. 14a. Tuomikoski, R. 1934: H 289.

Radula lindenbergiana Gott. ex Hartm.f. - 7e. Buch, H. 1911: H 290. Lindberg, S. O. 1874: H 291, HSOL 319.

Riccia beyrichiana Hampe ex Lehm. et Lindeb. - 5a. Lindberg, S. O. 1874: H 293, H-SOL 324. 7g. Lindberg, S. O. 1874: H 292, H-SOL 320-322. 13d. Huuskonen, A. J. 1937: H 294.

Scapania apiculata Spruce - Buch, H. 14a. Tuomikoski, R. 1934: H 296-299.

Scapania nemorea (L.) Grolle - Buch, H. 1953. 9b. Huuskonen, A. J. 1944: H 300, 301, OULU 412.

Trichocolea tomentella (Ehrh.) Dum. - 14a. Tuomikoski, R. 1934: H 302-304.

\section{ACKNOWLEDGEMENTS}

The study is a part of a project "Biodiversity of the northern coast of Lake Ladoga" (University of Helsinki, project no. 96978501), hosted by the Finnish Museum of Natural History. Financial support by the Ministry of the Environment is greatly acknowledged.

\section{LITERATURE CITED}

ANONYMOUS 1938. Suomen luonnontielteellisen alueen paikannimiluettelo. - Suomen Hyönteistieteellinen Aikakausikirja 4(2): 1-17.

BRANDT, A. 1933. Hiisjärven luonnonpuiston kasvillisuudesta. - Silva Fennica 32: 1-116.

BROTHERUS, V. F. 1913. Amblystegiella confervoides (Brid.) Loeske. - Meddelanden Soc. Fauna Flora Fennica 39: 62.

BUCH, H. 1938. Jungermannia tristis Nees frín Suistamo. - Memoranda Soc. Fauna Flora Fennica 14: 127.

CHYDENIUS, J. J. \& FURUHJELM, J. E. 1859. Berättelse öfver en naturhistorisk resa i Karelen, förtagen pí Sällskapets pro Fauna et Flora Fennica bekostnad. - Notiser Sällsk. Fauna Flora Fennica Förh. 4: 81-118.

HJELT, HJ. 1881. Anteckningar frín en botanisk resa I Karelen sommaren 1876. - Meddelanden Soc. Fauna Flora Fennica 6: 19-69.

IGNATOV, M. S \& AFONINA, O. M. 1992. Check-list of mosses of the former USSR. - Arctoa 1: 1-85.
JUNNILAINEN, R. 1977. The distribution of Aulacomnium androgynum and A. turgidum (Musci, Aulacomniaceae) in northwest Europe. - Memoranda Soc. Fauna Flora Fennica 53(2): 95-97.

KONSTANTINOVA, N. A., POTEMKIN, A. D. \& SCHLJAKOV, R. N. 1992. Check-list of the Hepatics and Anthocerotae of the former USSR. - Arctoa 1: 87-127.

KOPONEN, T. 1967. Eurynchium angustirete (Broth.) Kop. comb. n. (E. zetterstedtii Stoerm.) and its distribution pattern. - Memoranda Soc. Fauna Flora Fennica 43: 53-59.

KOTILAINEN,M.1927.Cirriphyllum vaucheri (Schimp.) Loesk. Et Fleisch. Ruskealassa. - Memoranda Soc. Fauna Flora Fennica 1: 329-331.

KOTILAINEN, M. 1929. Über das boreale Laubmoose-element in Ladoga-Karelien. Eine kausal-ökologische und floristische Studie. - Ann. Soc. Zool.-Bot. Fennicae Vanamo 11(1): 1-142, 16 maps. 
KOTILAINEN, M. 1931. Excursion nach Ladoga-Karelien (Laatokan Karjala) 8.-14. Juni 1930. - Memoranda Soc. Fauna Flora Fennica 7: 85-89.

KOTILAINEN, M. 1935. Anoectangium compactum Schwägr. Laatokan Karjalassa. - Memoranda Soc. Fauna Flora Fennica 11: 133-135.

KOTIRANTA, H. 1997. Red data book of eastern Fennoscandia.- Manuscript. Finnish Environment Institute.

LINDBERG, S. O. 1864. De speciebus Timmiae observationes. - Öfvers. K. Vet.-Akad. Förh. 6: 333-338.

MAKSIMOV, A. I. 1995. Mkhi (mosses). - In: Ivanter, E. V. \& Kuznetsov, O. L. (eds.) The Red data book of Karelia, Petrozavodsk, Karelia: 76-88.

NORRLIN, J. P. 1878. Symbolae ad floram Ladogensi-Karelicam. - Meddelanden Soc. Fauna Flora Fennica 2: 1-33.

NYLANDER, W. 1852a. Collectanea in Floram Karelicam. - Notiser sällsk. Fauna Flora Fennica Förh. 2: 109-181.

NYLANDER, W. 1852b. Collectanea in Floram Karelicam, continuatio. - Notiser Sällsk. Fauna Flora Fennica Förh. 2:183-201.

PESOLA, V. 1917. Kertomus kasvitieteellisestä tutkimusmatkasta Laatokan pohjoispuolisiin seutuihin kesänä 1914-1915. - Meddelanden Soc. Fauna Flora Fennica 43: 184-188.
PIIPPO, S. 1982. Epi phytic bryophytes as climatic indicators in Eastern Fennoscandia. - Acta Bot. Fennica 119: 1 -39.

TUOMIKOSKI, R. 1935. Hiisjärven luonnonpuiston sammalkasvisto. - Acta Soc. Fauna Flora Fennica 1:1-26.

TUOMIKOSKI, R. 1936. Über die Laubmoosarten Mnium affine, Mnium rugicum and Mnium seligeri. - Ann. Bot. Soc. Zool.-Bot. Fennicae Vanamo 6(5): 1-45.

TUOMIKOSKI, R. 1939. Lehtivihreätön saprofyyttinen maksasammal Cryptothallus mirabilis Malmb. - Luonnon ystävä 43: 11-14.

VAARAMA, A. 1936. Ricciocarpos natans Corda Suomessa. - Luonnon Ystävä 40: 49-56.

VAARAMA, A. 1939. Desmatodon latifolius (Hedw.) Bryol. eur.in Laatokka-Karelien. - Ann. Bot. Soc. Vanamo 11: 3.

VITIKAINEN, O. 1969. On the taxonomy and distribution of Grimmia anomala Hampe ex Schimper and G. hartmanii Schimper. - Acta Bot. Fennica 6(3): 236-242.

VOLKOVA, L. A. \& MAKSIMOV, A. I. 1993. Check-list of mosses of Karelia. - In: Elina, G. A. \& Volkov A. D. (eds.) Vegetation world of Karelia and the problems of it's protection. Petrozavodsk, Russian Academy of Sciences, Karelian research centre: 57-91. 